\title{
Oriented, 2-edge-colored, and 2-vertex-colored homomorphisms ${ }^{3}$ \\ Nazanin Movarraei ${ }^{1}$ and Pascal Ochem ${ }^{2}$, \\ ${ }^{1}$ Department of Mathematics, University of Pune, India \\ ${ }^{2}$ CNRS, LIRMM, Montpellier, France
}

\begin{abstract}
We show that the 2-edge-colored chromatic number of a class of simple graphs is bounded if and only if the acyclic chromatic number is bounded for this class. Recently, the CSP dichotomy conjecture has been reduced to the case of 2-edge-colored homomorphism and to the case of 2-vertex-colored homomorphism. Both reductions are rather long and follow the reduction to the case of oriented homomorphism in "Graphs and homomorphisms" by Hell and Nešetřil. We give an alternate proof of the case of 2 -vertex-colored homomorphism via a simple reduction from the case of 2-edge-colored homomorphism. Finally, we prove that deciding if the 2-edge-colored chromatic number of a 2-edge-colored graph is at most 4 is NP-complete, even if restricted to 2-connected subcubic bipartite planar graphs with arbitrarily large girth.
\end{abstract}

\section{Introduction}

A parameter $p$ defined on simple graphs is monotonous if $p\left(G^{\prime}\right) \leqslant p(G)$ for every subgraph $G^{\prime}$ of $G$. Given two monotonous parameters $p_{1}$ and $p_{2}$, we note $p_{1} \preceq p_{2}$ if there exists a function $f: \mathbb{N} \rightarrow \mathbb{N}$ such that $p_{1}(G) \leqslant f\left(p_{2}(G)\right)$ for every graph $G$. Moreover, $p_{1}$ and $p_{2}$ are equivalent if $p_{1} \preceq p_{2}$ and $p_{2} \preceq p_{1}$. We note $p_{1} \sim p_{2}$ if $p_{1}$ and $p_{2}$ are equivalent. For a graph class $\mathcal{G}$ and a monotonous parameter $p$, we define $p(\mathcal{G})=\max \{p(G) \mid G \in \mathcal{G}\}$. We consider as a graph class any subset of the class of all simple graphs, i.e., it is not necessarily closed under minor, induced subgraph, $\ldots$. The statement $p_{1} \sim p_{2}$ is equivalent to the statement that for every graph class $\mathcal{G}, p_{1}(\mathcal{G})=O(1)$ if and only if $p_{2}(\mathcal{G})=O(1)$.

In section 2 , we investigate the family $\mathcal{F}_{a}$ of graph parameters equivalent to the acyclic chromatic number $\chi_{a}$. We show that the 2-edge-colored chromatic number $\chi_{2}$ is in $\mathcal{F}_{a}$ by showing that $\chi_{2}$ is equivalent to the oriented chromatic number $\chi_{o}$, which is in $\mathcal{F}_{a}$. In Section 3, we show that the dichotomy conjecture for CSP can be reduced from the case of 2-edge-colored homomorphism to the case of 2-vertex-colored homomorphism. In Section 4, we show that 2-edge-colored homomorphism is NP-complete for 2-edge-colored planar graphs with arbitrarily large girth.

\section{Bounds on $\chi_{o}$ and $\chi_{2}$}

\section{$2.1 \quad$ Preliminaries}

A $k$-vertex is a vertex of degree $k$. An oriented graph is obtained from a simple graph by assigning an orientation to every edge. If $H_{o}$ is an oriented graph, then $A\left(H_{o}\right)$ denotes its set of arcs and $\overrightarrow{u v}$ denotes the arc from the vertex $u$ to the vertex $v$.

A 2-edge-colored graph is obtained from a simple graph by assigning a color or sign from the set $\{+,-\}$ to every edge. If $H_{2}$ is a , then $s g(u v)$ denotes the sign of the edge $u v$ and $E^{+}\left(H_{2}\right)$ (resp. $E^{-}\left(H_{2}\right)$ ) denotes its set of positive (resp. negative) edges. We thus have $u v \in E^{s g(u v)}\left(H_{2}\right)$. We also use $s g(z)$ to denote the sign of the number $z \in \mathbb{Z}^{*}$.

An $(n, m)$-mixed graph $M$ is a graph in which some pairs of vertices are linked by arcs and some are linked by edges such that all the arcs are colored with $n$ colors, all the edges are colored with $m$ colors, and the underlying graph of $M$ is simple. For $(n, m) \neq(0,0)$, the mixed chromatic number $\chi_{(n, m)}(M)$ is the minimum number of vertices of an $(n, m)$-mixed graph $T$ such that there exists a homomorphism from $M$ to $T$ that is compatible with the

\footnotetext{
${ }^{3}$ This work was partially supported by the ANR grant EGOS 12-JS02-002-01.
} 
colors of the arcs and the edges and with the orientation of the arcs. We define $\chi_{(n, m)}(G)$ as the maximum of $\chi_{(n, m)}(M)$ over the $(n, m)$-mixed graphs $M$ having $G$ as underlying simple graph. Thus, for a simple graph $G, \chi_{(0,1)}(G)=\chi(G)$ corresponds to the chromatic number of $G, \chi_{(1,0)}(G)=\chi_{o}(G)$ corresponds to the oriented chromatic number of $G$, and $\chi_{(0,2)}(G)=\chi_{2}(G)$ corresponds to the 2-edge-colored chromatic number of $G$.

The star chromatic number $\chi_{P_{4}}(G)$ corresponds to the minimum number of colors needed to color the vertices of $G$ such that no edge is monochromatic and no subgraph isomorphic to $P_{4}$ is bichromatic. The oriented chromatic index $\chi_{o}^{\prime}\left(G_{o}\right)$ corresponds to the oriented chromatic number of the line-digraph $L D\left(G_{o}\right)$ of the oriented graph $G_{o}$, that is, $\chi_{o}^{\prime}\left(G_{o}\right)=$ $\chi_{o}\left(L D\left(G_{o}\right)\right)$. The oriented chromatic index $\chi_{o}^{\prime}(G)$ of a simple graph $G$ is the maximum of $\chi_{o}^{\prime}\left(G_{o}\right)$ over all the orientations $G_{o}$ of $G$. The oriented chromatic index has been studied in $[14,15,16]$. The pushable chromatic number $\chi_{p}\left(G_{o}\right)$ is the minimum of $\chi_{o}\left(G_{o}^{\prime}\right)$ over the oriented graphs $G_{o}^{\prime}$ equivalent to $G_{o}$ under the push operation which reverses the arcs in an edge-cut of an oriented graph. The switch chromatic number $\chi_{s w}\left(G_{2}\right)$ is the minimum of $\chi_{o}\left(G_{2}^{\prime}\right)$ over the 2-edge-colored graphs $G_{2}^{\prime}$ equivalent to $G_{2}$ under the switch operation which changes the sign of the edges in an edge-cut of a 2-edge-colored graph. The definitions of $\chi_{p}$ and $\chi_{s w}$ similarly extend to simple graphs.

Theorem 1. For every graph $G, \chi_{o}(G) \leqslant \chi(G) \cdot \chi_{2}(G)$ and $\chi_{2}(G) \leqslant \chi(G) \cdot \chi_{0}(G)$.

We leave as an open problem whether the bounds in Theorem 1 are tight. We know the values of $\chi_{o}$ and $\chi_{2}$ for the class $\mathcal{T}_{g}$ (resp. $\mathcal{O}_{g}$ ) of partial 2-trees (resp. outerplanar graphs) with girth at least $g[10,12,16]$. In particular, we have:

$$
\begin{gathered}
8=\chi_{2}\left(\mathcal{T}_{5}\right)>\chi_{o}\left(\mathcal{T}_{5}\right)=6 \\
5=\chi_{2}\left(\mathcal{T}_{6}\right)<\chi_{o}\left(\mathcal{T}_{6}\right)=6 \\
9=\chi_{2}\left(\mathcal{O}_{3}\right)>\chi_{o}\left(\mathcal{O}_{3}\right)=7 \\
5=\chi_{2}\left(\mathcal{O}_{4}\right)<\chi_{o}\left(\mathcal{O}_{4}\right)=6
\end{gathered}
$$

This shows that there exist graphs $G$ such that $\chi_{2}(G)=\chi_{o}(G)+2$, or $\chi_{2}(G)=\chi_{o}(G)-1$.

\subsection{Proof of $\chi_{o}(G) \leqslant \chi(G) \cdot \chi_{2}(G)$}

Consider a proper coloring $c: V(G) \mapsto\{1, \ldots, \chi(G)\}$ of the simple graph $G$. Let $G_{o}$ be an orientation of $G$ such that $\chi_{o}\left(G_{o}\right)=\chi_{o}(G)$. Let $G_{2}$ be the 2-edge-colored graph with underlying simple graph $G$ such that for every $\operatorname{arc} \overrightarrow{u v} \in A\left(G_{o}\right)$, we have $u v \in E^{s g(c(v)-c(u))}\left(G_{2}\right)$. By definition of $\chi_{2}(G)$, there exists a 2-edge-colored homomorphism $h_{2}: G_{2} \rightarrow T_{2}$ such that $\left|V\left(T_{2}\right)\right| \leqslant \chi_{2}(G)$.

We construct the oriented graph $T_{o}$ as follows:

- $V\left(T_{o}\right)=[1, \ldots, \chi(G)] \times\left[1, \ldots,\left|V\left(T_{2}\right)\right|\right]$.

- If $1 \leqslant i \leqslant \chi(G), 1 \leqslant j \leqslant \chi(G), i \neq j$, and $m n \in E^{s g(j-i)}\left(T_{2}\right)$, then $\overrightarrow{(i, m)(j, n)} \in$ $A\left(T_{o}\right)$.

We define the mapping $h_{o}$ as the cartesian product of $c$ and $h_{2}$, that is, for every vertex $v \in V(G), h_{o}(v)=\left(c(v), h_{2}(v)\right)$. Let us check that $h_{o}$ defines an oriented homomorphism from $G_{o}$ to $T_{o}$. That is, for every arc $\overrightarrow{u v} \in A\left(G_{o}\right)$, we have $\overrightarrow{h_{o}(u) h_{o}(v)} \in A\left(T_{o}\right)$. By construction of $G_{2}$, we have $u v \in E^{s g(c(v)-c(u))}\left(G_{2}\right)$. Since $h_{2}$ preserves the sign of edges, we have $h_{2}(u) h_{2}(v) \in E^{s g(c(v)-c(u))}\left(T_{2}\right)$. Finally, by construction of $T_{o}$, this implies that $\overrightarrow{h_{o}(u) h_{o}(v)}=\overrightarrow{\left(c(u), h_{2}(u)\right)\left(c(v), h_{2}(v)\right)} \in A\left(T_{o}\right)$.

Thus, we have

$$
\chi_{o}(G)=\chi_{o}\left(G_{o}\right) \leqslant\left|V\left(T_{o}\right)\right|=\chi(G) \cdot\left|V\left(T_{2}\right)\right| \leqslant \chi(G) \cdot \chi_{2}(G) .
$$




\subsection{Proof of $\chi_{2}(G) \leqslant \chi(G) \cdot \chi_{o}(G)$}

The proof is similar to the previous one, since we essentially switch "oriented" and "2-edgecolored". Consider a proper coloring $c: V(G) \mapsto\{1, \ldots, \chi(G)\}$ of the simple graph $G$. Let $G_{2}$ be 2-edge-colored graph with underlying simple graph $G$ such that $\chi_{2}\left(G_{2}\right)=\chi_{2}(G)$. Let $G_{o}$ be the orientation of $G$ such that for every edge $u v \in E(G)$, we have $\overrightarrow{u v} \in A\left(G_{o}\right)$ if $u v \in E^{s g(c(v)-c(u))}\left(G_{2}\right)$. By definition of $\chi_{o}(G)$, there exists an oriented homomorphism $h_{o}: G_{o} \rightarrow T_{o}$ such that $\left|V\left(T_{o}\right)\right| \leqslant \chi_{o}(G)$.

We construct the 2-edge-colored graph $T_{2}$ as follows:

- $V\left(T_{2}\right)=[1, \ldots, \chi(G)] \times\left[1, \ldots,\left|V\left(T_{o}\right)\right|\right]$.

- If $1 \leqslant i \leqslant \chi(G), 1 \leqslant j \leqslant \chi(G), i \neq j$, and $\overrightarrow{m n} \in A\left(T_{o}\right)$, then $(i, m)(j, n) \in$ $E^{s g(j-i)}\left(T_{2}\right)$.

We define the mapping $h_{2}$ as the cartesian product of $c$ and $h_{o}$, that is, for every vertex $v \in$ $V(G), h_{2}(v)=\left(c(v), h_{o}(v)\right)$. Let us check that $h_{2}$ defines a 2-edge-colored homomorphism from $G_{2}$ to $T_{2}$. That is, every edge $u v$ in $G_{2}$ has the same sign as its image $h_{2}(u) h_{2}(v)$ in $T_{2}$.

By possibly interchanging $u$ and $v$, we can assume without loss of generality that $u v \in$ $E^{s g(c(v)-c(u))}\left(G_{2}\right)$. By construction of $G_{o}$, we have $\overrightarrow{u v} \in A\left(G_{o}\right)$. Since $h_{o}$ preserves the orientation of arcs, we have $\overrightarrow{h_{o}(u) h_{o}(v)} \in A\left(T_{o}\right)$. Finally, by construction of $T_{2}$, this implies that $h_{2}(u) h_{2}(v)=\left(c(u), h_{o}(u)\right)\left(c(v), h_{o}(v)\right) \in E^{s g(c(v)-c(u))}\left(T_{2}\right)$.

Thus, we have

$$
\chi_{2}(G)=\chi_{2}\left(G_{2}\right) \leqslant\left|V\left(T_{2}\right)\right|=\chi(G) \cdot\left|V\left(T_{o}\right)\right| \leqslant \chi(G) \cdot \chi_{o}(G) .
$$

\subsection{Parameters in $\mathcal{F}_{a}$}

As a Corollary of Theorem 1 and other results from the literature, we obtain the following.

Corollary 2. For every $(n, m) \in \mathbb{N}^{2} \backslash\{(0,0),(0,1)\}$, we have $\chi_{(n, m)} \in \mathcal{F}_{a}$. Moreover, we have $\left\{\chi_{P_{4}}, \chi_{o}^{\prime}, \chi_{p}, \chi_{s w}\right\} \subset \mathcal{F}_{a}$.

Proof. Notice that for $n^{\prime} \leqslant n$ and $m^{\prime} \leqslant m, \chi_{\left(n^{\prime}, m^{\prime}\right)} \leqslant \chi_{(n, m)}$. By Theorem 1 we have $\chi_{o}(G) \leqslant \chi(G) \cdot \chi_{2}(G) \leqslant\left(\chi_{2}(G)\right)^{2}$, which implies $\chi_{o} \preceq \chi_{2}$. That is

$$
\chi_{(1,0)} \preceq \chi_{(0,2)}
$$

We know that $\chi_{a} \leqslant\left(\chi_{o}\right)^{2}+\left(\chi_{o}\right)^{3+\left\lceil\log _{2} \log _{2} \chi_{o}\right\rceil}[8]$, which gives

$$
\chi_{a} \preceq \chi_{(1,0)}
$$

Also, for all $(n, m) \in \mathbb{N}^{2} \backslash\{(0,0)\}$, we have $\chi_{(n, m)} \leqslant \chi_{a} \cdot(2 n+m)^{\chi_{a}-1}$ [11], which gives

$$
\forall(n, m) \in \mathbb{N}^{2} \backslash\{(0,0)\}, \chi_{(n, m)} \preceq \chi_{a}
$$

If $n \geqslant 1$ and $m \geqslant 0$, then we use (2) and (3) to obtain

$$
\forall n \geqslant 1, \forall m \geqslant 0, \chi_{a} \preceq \chi_{(1,0)} \preceq \chi_{(n, m)} \preceq \chi_{a} .
$$

This implies

$$
\forall n \geqslant 1, \forall m \geqslant 0, \chi_{(n, m)} \in \mathcal{F}_{a} .
$$

Also, if $n \geqslant 0$ and $m \geqslant 2$, then we use (1) to obtain

$$
\forall n \geqslant 0, \forall m \geqslant 2, \chi_{a} \preceq \chi_{(1,0)} \preceq \chi_{(0,2)} \preceq \chi_{(n, m)} \preceq \chi_{a} .
$$

This implies

$$
\forall n \geqslant 0, \forall m \geqslant 2, \chi_{(n, m)} \in \mathcal{F}_{a} .
$$

Together, (5) and (7) prove the first statement of Corollary 2.

The second statement follows from known bounds from the literature. 
- $\chi_{a} \leqslant \chi_{P_{4}} \leqslant \chi_{a} \cdot\left(2 \chi_{a}-1\right)[1]$, so $\chi_{P_{4}} \sim \chi_{a}$ and thus $\chi_{P_{4}} \in \mathcal{F}_{a}$.

- $\chi_{o}^{\prime} \leqslant \chi_{o} \leqslant\left(\left\lfloor\chi_{o}^{\prime} / 2\right\rfloor+2\right) \cdot 2^{\left\lfloor\left(\chi_{o}^{\prime}-1\right) / 2\right\rfloor}[14]$, so $\chi_{o}^{\prime} \sim \chi_{o}$ and thus $\chi_{o}^{\prime} \in \mathcal{F}_{a}$.

- $\chi_{p} \leqslant \chi_{o} \leqslant 2 \chi_{p}[7]$, so $\chi_{p} \sim \chi_{o}$ and thus $\chi_{p} \in \mathcal{F}_{a}$.

- $\chi_{s w} \leqslant \chi_{2} \leqslant 2 \chi_{s w}[13]$, so $\chi_{s w} \sim \chi_{2}$ and thus $\chi_{s w} \in \mathcal{F}_{a}$.

\section{Dichotomy conjecture for 2-vertex-colored homomor- phism}

The dichotomy result of Hell and Nešetřil for simple graph homomorphism [6] states that homomorphism to $H$ is decidable in polynomial time if $H$ is bipartite and is NP-complete otherwise. The well known dichotomy conjecture of Feder and Vardi [4] states that for every family $\Gamma$ of constraints, $\operatorname{CSP}(\Gamma)$ is either testable in polynomial time or NP-complete. They established that it is sufficient to settle the dichotomy conjecture when $\Gamma$ contains a single binary relation, i.e. is a directed graph $H$. We say that a 2-edge-colored graph is alternating if it maps to the infinite path $P_{e}$ such that consecutive edges have distinct signs. We say that a 2-vertex-colored graph is alternating if it maps to the infinite path $P_{v}$ such that every vertex is adjacent to one positive vertex and one negative vertex. Recently, the dichotomy conjecture has been shown to be equivalent to the case of homomorphism to 2-edge-colored alternating graphs [3] and to the case of homomorphism to 2-vertex-colored alternating graphs [5]. In this section, we give an alternate proof for the case of 2-vertex-colored homomorphism via a simple reduction from the case of 2-edge-colored homomorphism.

Let $F$ be the function which associates to a 2-edge-colored graph $G_{e}$ the 2-vertex-colored graph $G_{v}=F\left(G_{e}\right)$ obtained as follows:

- For every vertex in $G_{e}$, we put a positive vertex in $G_{v}$.

- For every positive edge $x y$ in $G_{e}$, we put an edge in $G_{v}$ between the vertices corresponding to $x$ and $y$.

- For every negative edge $x y$ in $G_{e}$, we put a path $x^{\prime} a b y^{\prime}$ in $G_{v}$ such that $w^{\prime}$ and $y^{\prime}$ correspond to $x$ and $y$ and such that $a$ and $b$ are negative.

Theorem 3. For every 2-edge-colored alternating graph $T_{e}$, homomorphism to $T_{e}$ is polynomially equivalent to homomorphism to the 2-vertex-colored alternating graph $F\left(T_{e}\right)$.

Proof. Notice that since $P_{v}=F\left(P_{e}\right)$, the $F$-image of a 2-edge-colored alternating graph is indeed a 2-vertex-colored alternating graph. By construction, the $F$-image of a 2-edgecolored graph is such that every negative vertex has exactly one positive neighbor and exactly one negative neighbor. For every vertex $x$ in 2-edge-colored $G_{e}$, we denote by $x^{\prime}$ the corresponding positive vertex in $G_{v}=F\left(G_{e}\right)$.

First, we show that a 2-edge-colored graph $G_{e}$ maps to $T_{e}$ if and only if $G_{v}=F\left(G_{e}\right)$ maps to $T_{v}=F\left(T_{e}\right)$. It is straightforward to see that if $G_{e}$ maps to $T_{e}$, then $G_{v}$ maps to $T_{v}$. Suppose that $G_{v}$ admits a 2-vertex-colored homomorphism $h$ to $T_{v}$. Let us check that the restriction $h^{+}$of $h$ to the positive vertices of $G_{v}$ gives a 2-edge-colored homomorphism from $G_{e}$ to $T_{e}$. For every positive edge $x y$ in $G_{e}$, the edge $x^{\prime} y^{\prime}$ in $G_{v}$ linking the positive vertices $x^{\prime}$ and $y^{\prime}$ maps in $T(v)$ to the edge $h\left(x^{\prime}\right) h\left(y^{\prime}\right)=\left(h^{+}(x)\right)^{\prime}\left(h^{+}(y)\right)^{\prime}$ in $G_{v}$ linking the positive vertices $h\left(x^{\prime}\right)$ and $h\left(y^{\prime}\right)$. For every negative edge $x y$ in $G_{e}$, the path $x^{\prime} a b y^{\prime}$ in $G_{v}$ linking the positive vertices $x^{\prime}$ and $y^{\prime}$ must map in $T(v)$ to a path $h\left(x^{\prime}\right) h(a) h(b) h\left(y^{\prime}\right)$ in $G_{v}$ linking the positive vertices $h\left(x^{\prime}\right)=\left(h^{+}(x)\right)^{\prime}$ and $h\left(y^{\prime}\right)=\left(h^{+}(y)\right)^{\prime}$.

Now, given a 2-vertex-colored graph $J_{v}$, we construct a 2-edge-colored graph $J_{e}$ such that $J_{e}$ maps to $T_{e}$ if and only if $J_{v}$ maps to $T_{v}=F\left(T_{e}\right)$, for every 2-edge-colored bipartite 
graph $T_{e}$. We test whether $J_{v}$ is alternating. Testing whether a 2-vertex-colored graph is alternating is polynomial-time solvable:

- Answer no if contains a loop or an odd cycle.

- For every vertex $v$, identify all the positive neighbors of $v$ into one vertex and identify all the positive neighbors of $v$ into one vertex.

- Answer yes if and only if the obtained graph is a forest (i.e., maps to $P_{v}$ ).

If $J_{v}$ is not alternating, then $J_{v}$ does not map to $T_{v}$ and we assign to $T_{e}$ the triangle with 3 positive edges, so that $J_{e}$ does not map to $T_{e}$. Otherwise, we modify $J_{v}$ as follows:

1. For every negative vertex $u$, we identify all the positive (resp. negative) neighbors of $u$ into one vertex.

2. For every negative vertex $u$ that has no negative neighbor, we add a negative vertex adjacent to $u$.

3. For every negative vertex $u$ that has no positive neighbor, we add a positive vertex adjacent to $u$.

4. As long as there exists two distinct paths $x a b y$ and $x a^{\prime} b^{\prime} y$ such that $x$ and $y$ are positive and $a, b, a^{\prime}$, and $b^{\prime}$ are negative, remove $a^{\prime}$ and $b^{\prime}$.

We thus obtain an alternating graph $J_{v}^{*}$. Since every negative vertex in $T_{v}$ has exactly one positive neighbor and one negative neighbor, $J_{v}^{*}$ maps to $T_{v}$ if and only if $J_{v}$ maps to $T_{v}$. Moreover, every negative vertex of $J_{v}^{*}$ has exactly one positive neighbor and one negative neighbor. We obtain $J_{e}$ from $J_{v}^{*}$ by smoothing the negative vertices, such that the original edges in $J_{e}$ are positive in $J_{v}$ and the smoothed edges are negative. Notice that $J_{e}$ does not contain multiple negative edges. Thus, up to isomorphism, $J_{e}$ is the unique 2-edge-colored graph such that $J_{v}=F\left(J_{e}\right)$. By the previous arguments, $J_{e}$ maps to $T_{e}$ if and only if $J_{v}$ maps to $T_{v}$.

\section{Complexity of 2-edge-colored homomorphism}

In this section, we investigate the case of 2-edge-colored homomorphism. A 2-edge-colored graph is a core if it does not admit a homomorphism to one of its proper (induced) subgraphs. If a 2-edge-colored core $H$ contains a monochromatic odd cycle of sign $s$, then the homomorphism to $H$ restricted to input graphs containing only edges of sign $s$ is NP-complete [6]. Thus, we focus on 2-edge-colored cores $H$ such that the monochromatic subgraphs of $H$ are bipartite. Cores with such properties are said interesting. It is easy to see that homomorphism to an interesting core with at most 3 vertices is polynomial. The next result shows that homomorphism to the interesting core $T_{4}$ with 4 vertices depicted in Figure 1 is NP-complete.

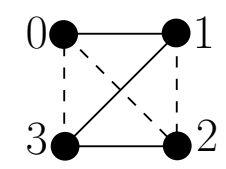

Figure 1: The 2-edge-colored graph $T_{4}$.

Theorem 4. Let $k$ be a fixed integer. Then 2-edge-colored homomorphism to $T_{4}$ is NPcomplete even if restricted to 2-connected planar graphs with maximum degree 3 such that the distance between two 3-vertices is even and at least $k$. 
Proof. 2-edge-colored homomorphism in general is clearly in NP. We reduce the NP-complete problem [9] PLANAR $(3, \leqslant 4)$-SAT. This variant of SAT is such that:

- every clause contains exactly 3 literals,

- every variable occurs in at most 4 clauses,

- the variable-clause incidence graph is planar.

Given an instance $I$ of PLANAR $(3, \leqslant 4)$-SAT, we will construct a 2-edge-colored graph $G$ corresponding to $I$. Consider the mappings $m_{i}: x \rightarrow(x+i)(\bmod 4)$. We partition the vertex set of $T_{4}$ into two levels. One level contains vertices 0 and 1 and the other level contains vertices 2 and 3 . Then $m_{0}$ is the identity, $m_{1}$ and $m_{3}$ are an anti-automorphism, that is, a mapping that maps every edge to an edge of opposite sign, and $m_{2}$ is an automorphism of $T_{4}$ that swaps the levels. We say that colors, i.e., vertices of $T_{4}$, are opposite if they are on the same level and are distinct. That is, 0 and 1 are opposite and 2 and 3 are opposite. For a color $x$, we denote by $\bar{x}$ the opposite color.

A $4 k$-path is a path of length $\ell$ such that consecutive edges have distinct signs, $\ell \geqslant 4$, and $\ell \equiv 0(\bmod 4)$. The positive extremity (resp. negative extremity) of a $4 k$-path is its 1 -vertex incident to a positive (resp. negative) edge. A $4 k$-cycle is obtained by identifying the the positive and the negative extremities of a $4 k$-path. In a $4 k$-cycle in $G$, the distance between every two 3 -vertices on the $4 k$-cycle is $0(\bmod 4)$. Thus, a $4 k$-cycle has exactly four distinct homomorphisms to $T_{4}$ depending on the common color of its 3 -vertices. We define the color of a $4 k$-cycle as the color of its 3 -vertices. In the plane representation of $G$, every $4 k$-cycle has an empty interior.

We construct $G$ as follows.

1. For every variable of $I$, we put a $4 k$-cycle (the variable gadget) in $G$.

2. For every clause of $I$, we put one copy of the clause gadget depicted on the left of Figure 2. Every 1-vertex in a clause gadget corresponds to an occurrence of a literal in the clause. Such a vertex is called a port.

3. Whenever a clause contains a positive literal of a variable, we take one copy of a $4 k$ path, we identify the positive extremity of the $4 k$-path to an appropriate vertex on the $4 k$-cycle of the variable gadget, and we identify the negative extremity of the $4 k$-path to the corresponding port in the clause gadget.

4. Whenever a clause contains a negative literal of a variable, we take one copy of a $4 k$-cycle and place it between the variable gadget and the clause gadget. We link the variable gadget to the new $4 k$-cycle using four paths, as depicted in Figure 3 . Each of these four paths consists in a $4 k$-path and two additional edges, so it can be arbitrarily long. These paths force that the colors of the two $4 k$-cycles are opposite. The paths $m m^{\prime}$ and $n n^{\prime}$ force that the colors of the $4 k$-cycles belong to the same level. The paths $o o^{\prime}$ and $p p^{\prime}$ force that the colors of the $4 k$-cycles are distinct. Then we take one copy of a $4 k$-path, we identify the positive extremity of the $4 k$-path to an appropriate vertex on the new $4 k$-cycle, and we identify the negative extremity of the $4 k$-path to the corresponding port in the clause gadget.

5. Whenever two variables appear in the same clause, their clause gadgets appear consecutively on a face of $G$. We link the $4 k$-cycles of the two clause gadgets using only the paths $m m^{\prime}$ and $n n^{\prime}$ in Figure 3 and preserve planarity. As already discussed, these paths ensure that the colors of the two clause gadgets are on the same level. By connectivity, the colors of all the clause gadgets in $G$ are on the same level. Without loss of generality, this common level contains the colors 0 and 1. 

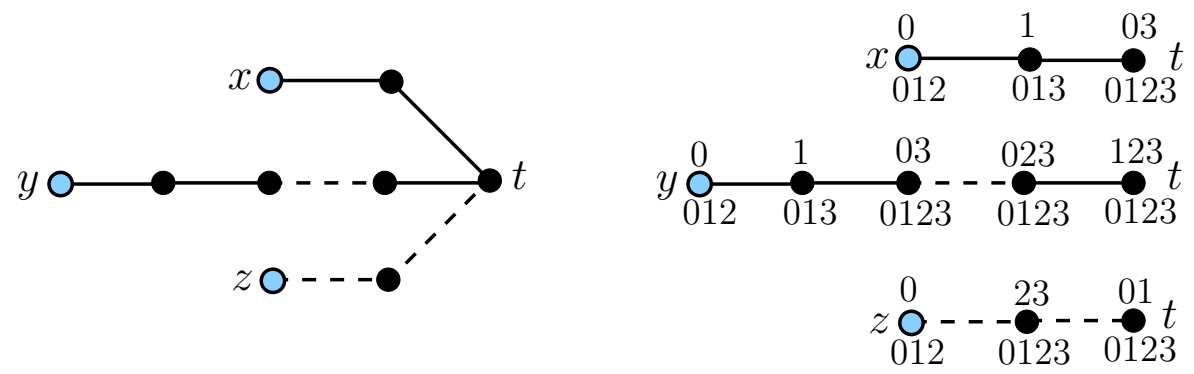

Figure 2: The clause gadget (left) and its coloring properties (right)

Notice that every occurrence of a variable in a clause is associated to a $4 k$-cycle in $G$ : the $4 k$-cycle of the variable gadget for a positive literal and the $4 k$-cycle added in step 4 for a negative literal. Also, every occurrence of a variable in a clause is associated to a $4 k$-path whose positive extremity is in the associated $4 k$-cycle and whose negative extremity is the port of the clause gadget. Figure 4 shows the possible color propagation along the vertices of a $4 k$-path depending on the color of the positive extremity. This leads to the following notion of boolean value, depending on the extremity of the associated $4 k$-path. The boolean value true of a literal is associated to the set of colors $\{1\}$ for the vertex in the $4 k$-cycle and is associated to $\{0,1,2\}$ for the port of the clause gadget. The boolean value false of a literal is associated to the set of colors $\{0\}$ for the vertex in the $4 k$-cycle and is associated to $\{0\}$ for the port of the clause gadget.

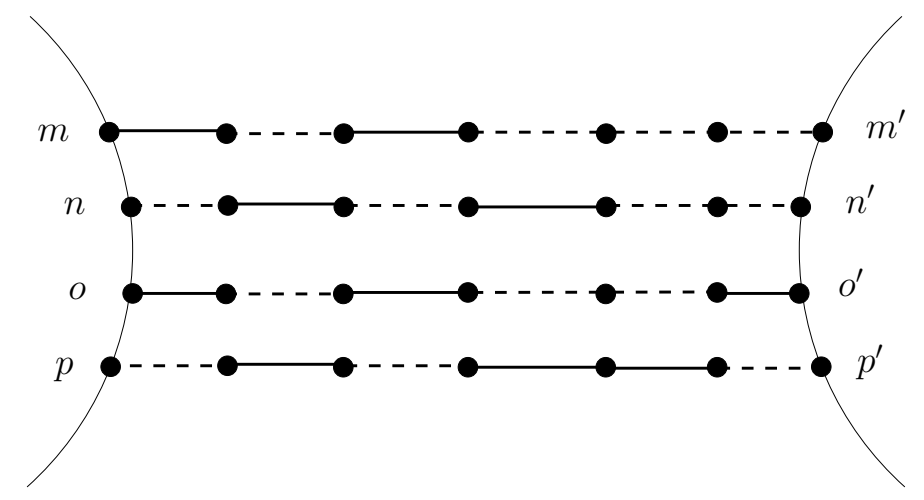

Figure 3: Forcing opposite colors on two $4 k$-cycles.

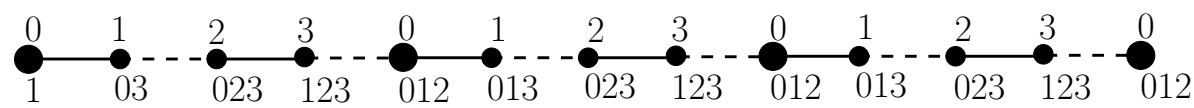

Figure 4: Color propagation along a $4 k$-path.

Now let us assume that the ports of a clause gadget are precolored according to their corresponding literal.

On the right of Figure 2, we give the possible color extensions of the three paths of the clause gadget, both in the case of a true literal (below the path) and in the case of a false literal (above the path). If a clause is satisfied, then at least one of its literal is true and the precoloring can be extended to a $T_{4}$-coloring of the vertex gadget. Indeed, if the literal corresponding to $x$ (resp. $y, z$ ) is true, then the precoloring can be extended such that 
$t$ is colored 1 (resp. 0,3 ). If it is not satisfied, then the precoloring cannot be extended to a $T_{4}$-coloring of the clause gadget. Indeed, if we have $c(x)=c(y)=c(z)=0$, then $c(t) \notin\{1,2\}$ because $c(x)=0, c(t) \neq 0$ because $c(y)=0$, and $c(t) \notin\{2,3\}$ because $c(z)=0$. So $c(t) \notin\{0,1,2,3\}$ and the clause gadget is not $T_{4}$-colorable.

Finally, notice that between every two 3 -vertices, there exists a $4 k$-path and its length can be arbitrarily large.

The restrictions on the input graph $G$ in Theorem 4 imply that $G$ is bipartite, all the 3 -vertices belong to the same part, and $G$ has arbitrarily large girth. Moreover, we see that $G$ contains no monochromatic path of length 5 and no vertex incident to three edges of the same sign.

Notice that the input graphs in the proof of Theorem 4 contain both a $4 k$-cycle and a cycle containing an odd number of positive edges and an odd number of negative edges, e.g., the cyle containing $n, n^{\prime}, o^{\prime}$, and $o$ in Figure 3. These cycles imply that the input graphs do not map to any 2 -edge-colored graph with at most 4 vertices other than $T_{4}$. We thus have

Corollary 5. Deciding $\chi_{2} \leqslant 4$ is NP-complete for the same class of 2-edge colored graphs as in Theorem 4.

\section{References}

[1] M. O. Albertson, G. G. Chappell, H. A. Kierstead, A. Kündgen, and R. Ramamurthi. Coloring with no 2-Colored $P_{4}$ 's. The Electronic Journal of Combinatorics 11(1), \#R26, 2004.

[2] M. de Berg and A. Khosravi. Optimal binary space partitions in the plane. LNCS 6196, 216-250, 2010.

[3] R. Brewster, F. Foucaud, P. Hell, and R. Naserasr. The complexity of signed graph and 2-edge-coloured graph homomorphisms. arXiv:1510.05502

[4] T. Feder and M. Y. Vardi The computational structure of monotone monadic (SNP) and constraint satisfaction: A study through datalog and group theory SIAM Journal of Computing, 28, 57-105, 1998.

[5] F. Foucaud, A. Harutyunyan, P. Hell, S. Legay, Y. Manoussakis, R. Naserasr, and P. Valicov. Tropical homomorphism and its complexity. Submitted

[6] P. Hell, J. Nešetřil. On the complexity of $H$-coloring. J. Combin. Theory, Ser. B 48, 92-110, 1990.

[7] W. F. Klostermeyer and G. MacGillivray. Homomorphisms and oriented colorings of equivalence classes of oriented graphs. Discrete Mathematics 274(1-3), 161-172, 2004.

[8] A.V. Kostochka, É. Sopena, and X. Zhu. Acyclic and oriented chromatic numbers of graphs. J. Graph Theory 24(4), 331-340, 1997.

[9] J. Kratochvil. A Special Planar Satisfiability Problem and a Consequence of its NPCompleteness. Discrete Appl. Math. 52, 233-252, 1994.

[10] A. Montejano, P. Ochem, A. Pinlou, A. Raspaud, and É. Sopena. Homomorphisms of 2-edge-colored graphs Discrete Applied Mathematics 158(12), 1365-1379, 2010.

[11] J. Nešetřil and A. Raspaud. Colored homomorphisms of colored mixed graphs $J$. Combin. Theory, Ser. B, 80(1), 147-155, 2000. 
[12] P. Ochem and A. Pinlou. Oriented colorings of partial 2-trees. Information Processing Letters 108, 82-86, 2008.

[13] P. Ochem, A. Pinlou, and S. Sen. Homomorphisms of signed planar graphs. arXiv: 1401.3308

[14] P. Ochem, A. Pinlou, and É. Sopena. On the oriented chromatic index of oriented graphs. J. Graph Theory 57(4), 313-332, 2008.

[15] A. Pinlou. On oriented arc-coloring of subcubic graphs. Electronic journal of Combinatorics 13, \#R69, 2006.

[16] A. Pinlou and É. Sopena. Oriented vertex and arc colorings of outerplanar graphs. Information Processing Letters 100(3), 97-104, 2006. 